\section{Patient compliance with deep vein thrombosis prophylaxis after total hip and total knee arthroplasty}

\author{
Daniel H. Wiznia, Nishwant Swami, \\ Jenny Nguyen, Eric Musonza, Chris \\ Lynch, David Gibson, Richard Pelker
}

Department of Orthopedics and Rehabilitation, Yale University School of Medicine, New Haven, CT, USA

\begin{abstract}
Even though the Centers for Medicare and Medicaid Services is penalizing hospitals for readmissions, and postoperative prophylaxis has demonstrated reduced complications associated with deep vein thrombosis (DVT), few studies have examined patient compliance with (DVT) prophylaxis at home. A survey querying DVT prophylaxis management and adherence was administered to patients who were within the one to three-month postoperative period after a total knee or total hip replacement. A total of 103 patients completed the survey. A considerable number of patients $(17.0 \%)$ were non-adherent to DVT prophylaxis. Patients had a lower understanding of the side effects of their DVT prophylaxis, with $30 \%$ responding that they had a poor to simple understanding. There is a high rate of non-compliance and there is a poor understanding of complications related to venous thromboembolism prophylaxis. As the population undergoing arthroplasties grows, this study demonstrates the importance of investigating the role of medication adherence in the rate of postoperative DVT.
\end{abstract}

\section{Introduction}

Post-operative total joint patients are at risk for venous thromboembolism (VTE) and pulmonary embolisms (PE), and pharmacologic deep vein thrombosis (DVT) prophylaxis and/or mechanical compression devices have become the standard of care..$^{1,2}$ Postoperative prophylaxis has demonstrated reduced complications associated with deep vein thrombosis (DVT), and the benefits of prophylaxis have been demonstrated post discharge. ${ }^{3-5}$ Patient adherence to DVT prophylaxis has become even more critical given that the Center for Medicare and Medicaid Services (CMS) defines VTEs as never events, and Medicare will not cover the additional costs for managing these complications. ${ }^{6}$ To prevent VTEs, surgeons place arthroplasty patients on pharmacologic DVT prophylaxis postoperatively and discharge their patients with instructions to continue taking DVT prophylaxis medication for a total of 10 to 30 days. $^{2}$

While most patients are discharged on pharmacologic DVT prophylaxis, surgeons should not assume that their patients are actually taking their medication. There is mounting evidence that a small minority of postoperative patients are not complying with their doctors' orders upon discharge. ${ }^{7-9}$ In the literature, compliance with home DVT prophylaxis for total hip and knee patients ranges from $88-90 \% .^{10,11}$ During a quality improvement review of our arthroplasty service, a focus was placed on comparing our patient DVT compliance to the above described studies.

While $100 \%$ compliance may not be truly obtainable, it is an aspiration that is in the best interest of our patients. As CMS includes total joint arthroplasty re-admissions as a factor in determining payment reductions, patient compliance with DVT prophylaxis can have a major effect on hospital system fiscal health. The purpose of our study was to evaluate our postoperative THA and TKA patients' DVT prophylaxis compliance and to examine which factors influence compliance. We also wanted to examine the incidence of bleeds as a consequence of DVT prophylaxis. We hypothesize that patients will be more compliant with oral medication than with injectables.

\section{Materials and Methods}

This prospective study used an observational, longitudinal design. Between June 2014 and December 2014, patients within the 1 to 3-month post-operative period after a total hip or knee replacement were enrolled in six surgeons' joint replacement clinics. A paper-based survey was administered to patients while they were waiting to be seen in their clinic exam room. Only $20 \%$ of eligible patients were enrolled due to staffing/clerical limitations. At the time of this study, each surgeon had an individual preference regarding their patients' DVT prophylaxis medication. Three surgeons preferred patients without risk factors for DVTs receive $325 \mathrm{mg}$ of aspirin twice a day (25 patients), and patients with risk factors for DVTs receive warfarin with an INR goal of 1.8-2.2 (13 patients). Two surgeons preferred to discharge all patients on warfarin, with an INR goal of 1.8-2.2 (52 patients). One surgeon preferred to dis-
Correspondence: Daniel Wiznia, Department of Orthopedics and Rehabilitation, Yale University School of Medicine, 47 College Street, 1st Floor, New Haven, CT 06510, USA.

Tel.: +1.203.785.2579

E-mail: daniel.wiznia@yale.edu

Key words: medication compliance, medication adherence, DVT prophylaxis, total knee replacement, total hip replacement.

Contributions: DW designed the study, prepared the instruments, oversaw the IRB process, oversaw the data collection, conducted statistical analyses, interpreted the results and prepared of the manuscript. NS conducted the data collection, interpreted the results, and prepared of the manuscript. JN conducted the data collection, interpreted the results, and prepared the manuscript. EM conducted the data collection, interpreted the results, and prepared the manuscript. CL designed the study, oversaw the data collection, interpreted the results and prepared and revised the manuscript. DG designed the study, oversaw the data collection, interpreted the results and prepared and revised the manuscript. RP designed the study, oversaw the data collection, interpreted the results and prepared and revised the manuscript.

Conflict of interest: the authors declare no potential conflict of interest.

Funding: The Department of Orthopedics and Rehabilitation, Yale University School of Medicine provided a research $\$ 1000$ grant. None of the authors have any conflict of interest.

Received for publication: 20 October 2018. Accepted for publication: 7 june 2019 .

This work is licensed under a Creative Commons Attribution-NonCommercial 4.0 International License (CC BY-NC 4.0).

(C) Copyright D.H. Wiznia et al., 2019

Licensee PAGEPress, Italy

Hematology Reports 2019; 11:7914

doi:10.4081/hr.2019.7914

charge all patients on prophylactic doses of enoxaparin ( 7 patients). Four patients had been maintained on Pradaxa (dabigatran) or Xarelto (rivaroxaban) prior to surgery, and these patients were restarted on this regimen on post-operative day two. Each patient received a set of written discharge instructions that explained the importance of DVT prophylaxis. In addition, patients received instruction on the importance of DVT prophylaxis from nursing prior to discharge. Patients also received a list of their prescribed medications. All patients were to maintain prophylaxis for 28 days. Patients 
discharged on enoxaparin received nursing education on how to self-administer injections. The survey (Appendix) queried for demographic information (age, sex, marital status, occupation), details of the surgery (approach, laterality, discharge location, readmission), DVT prophylaxis management (type of medication, risk for DVTs, deviations from recommended regimen, understanding of regimen), and characteristics of care once they returned home (understanding of discharge instructions, identity and number of caretakers). Nonadherence to DVT prophylaxis was defined as missing at least one dose or deviating from the prescribed dose.

Statistical analysis was performed using SPSS version 21 (SPSS, Inc, Chicago, IL). Chi-square test or Fisher's exact test was used to analyze differences in categorical variables. Missing data was assumed to be missing at random. Unless otherwise stated, all statistical testing was performed with an alpha-level of 0.05 .

The study was submitted to and approved by our Institutional Review Board office.

\section{Results}

Between June 2014 and December

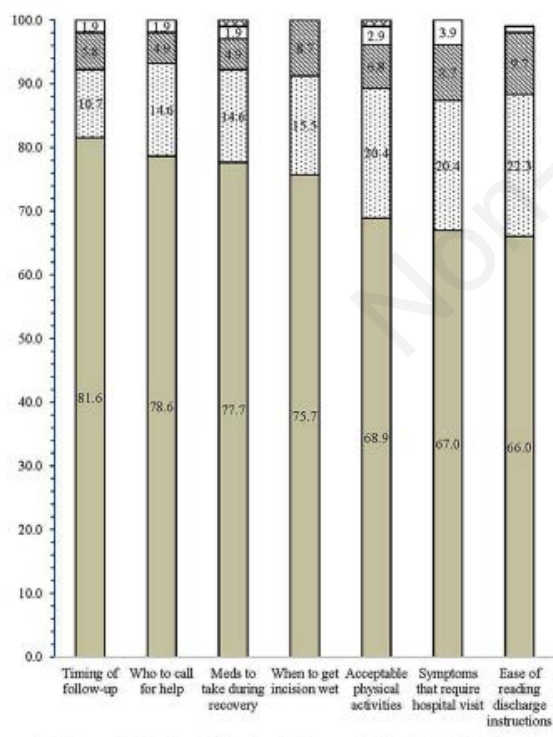

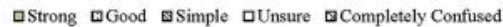

Figure 1. Patient understanding of discharge instructions. Patient responses regarding their understanding of discharge instructions versus percent of patients. Responses were collected with a 5-point Likert scale (Strong, good, simple, unsure, completely confused).
2014, a total of 103 patients completed the questionnaire. The mean age (Table 1) of the patients was 67 years, with $43 \%$ male and $83 \%$ white. Patients who were married or in relationships comprised $57 \%$ of the sample, with employed patients making up $36 \%$. Regarding surgical parameters, 50 patients had a total hip replacement and 53 patients had a total knee replacement (Table 1). A small percentage of patients stated that they had difficulty understanding discharge instructions (Figure 1). 21.4\% of patients were discharged home without a family member or friend caretaker (Table 2).

The most widely used DVT prophylaxis medications after surgery were warfarin (64.4\%) and aspirin (24.7\%), with enoxaparin $(6.9 \%)$ and dabigatran/rivaroxaban (4\%) less frequently used (Table 2). $17.0 \%$ of patients were non-adherent to DVT prophylaxis medications, of which $13.9 \%$ missed at least one dose and 3.1\% took more medicine than prescribed. Type of medication, route of drug delivery (injection or oral), cost, and occupation (employed/non-employed, manual/nonmanual, full/part time) did not have a statistically significant effect on medication compliance. Figure 2 demonstrates the level of understanding that patients had regarding their DVT prophylaxis instructions. The majority of patients responded that they had a strong understanding of how much med-

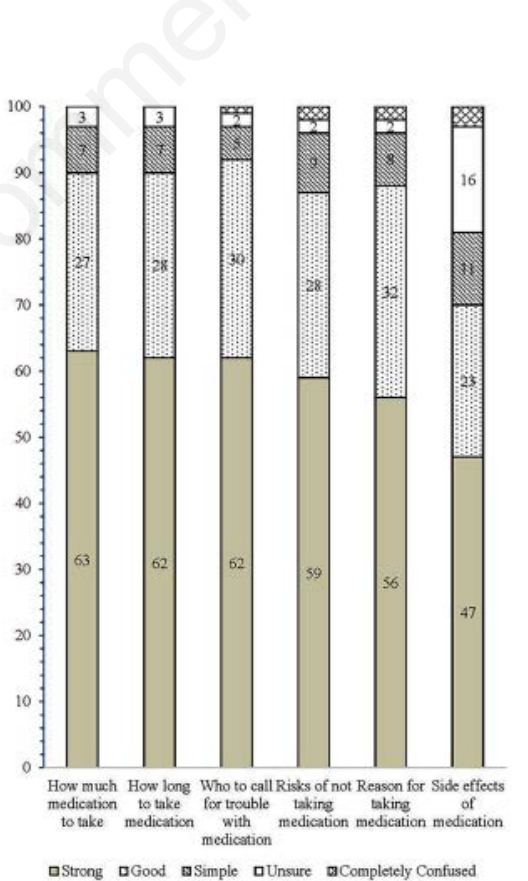

Figure 2. Patient understanding of DVT prophylaxis regimen. Patient responses regarding their understanding of their DVT prophylaxis regimen versus percent of patients. Responses were collected with a 5-point Likert scale (Strong, good, simple, unsure, completely confused). ication to take, how long to take the medication, who to call for trouble with medication, risks of not taking the medication, and the reason for taking the medication. However, patients had a lower understanding of the side effects of the medication, with only $47 \%$ responding that they had a strong understanding and $16 \%$ responding that they were unsure.

In terms of complications, $14.9 \%$ of patients required a work-up for a DVT/PE with an ultrasound or CT scan, which was significantly higher in patients who were single (23.8\% vs $8.6 \%, \mathrm{P}=0.036)$ (Table 3) and in patients who were discharged on

Table 1. Demographic information and surgical characteristic.

Variables $\quad$ Frequency $(\mathrm{N}=103)$
Demographic details

\section{Patient age (mean)}

67

\begin{tabular}{cc} 
Sex & \\
$M$ & 44 \\
$F$ & 59 \\
\hline
\end{tabular}

Race

$\begin{array}{lc}\text { White } & 87 \\ \text { Black } & 13 \\ \text { Asian } & 1 \\ \text { Hispanic } & 2\end{array}$

\begin{tabular}{lc} 
Marital status & \\
Married & 60 \\
Single & 43 \\
\hline Occupation & \\
Working & 37 \\
Not working & 66 \\
\multicolumn{2}{c}{ Surgical parameters }
\end{tabular}

\begin{tabular}{ll} 
Joint replaced & \\
Hip & 50 \\
Knee & 53 \\
Side replaced & \\
Unilateral & 92 \\
Bilateral & 11 \\
\hline Location after discharge & \\
Home & 50 \\
Rehabilitation facility & 53 \\
\hline
\end{tabular}

Table 2. Patient characteristics that influenced outcomes. Profile of married $v s$. not married patients.

\begin{tabular}{lc} 
Variables & Frequency \\
Number of people at home & \\
available to help (N=103) & \\
0 & 22 \\
1 & 52 \\
2 & 15 \\
$>2$ & 14 \\
DVT prophylaxis & \\
Aspirin & 25 \\
Warfarin & 65 \\
noxaparin & 7 \\
Dabigatran/rivaroxaban & 4 \\
\hline
\end{tabular}


warfarin $(20.6 \%$ vs $3.1 \%, \mathrm{P}=0.023)$ (Table 4). The operating surgeon conducted the work-up when patients presented to their clinics with concerning exams. In some cases, these work-ups were led by a medical team upon re-admission to the hospital. No patients suffered draining or a postoperative bleed.

\section{Discussion}

Post-operative compliance with DVT prophylaxis is crucial to overall operative success. Maintaining patients on postoperative DVT prophylaxis at home is a challenge, given that many hospitals struggle to maintain inpatients on adequate prophylaxis. ${ }^{12,13}$ Initiatives undertaken in the hospital setting, such as physician education, use of clinical practice guidelines, use of order sets with VTE prophylaxis options, progress notes with DVT prophylaxis included in a checklist, and electronic alerts to prescribers ${ }^{12,14-19}$ have increased physician use of prophylaxis and reduced rates of in hospital DVTs.

Previous studies that have examined TKA and THA patients' home compliance with DVT prophylaxis found $88-90 \% 10,11,20$ of patients adhered to their medication regimens. Studies examining the compliance of patients at high risk for DVTs demonstrated similar compliance patterns. ${ }^{7,9}$

Regarding reasons of noncompliance, the literature demonstrates that most patients forget to take 1-2 doses, ${ }^{11}$ have difficulty purchasing the medication, lack proper instructions or lack someone available to administer the injection. ${ }^{10}$ The literature characterizes patients who are compliant to be younger, employed and with a higher degree of education. $7,8,21$

In our study, $17.0 \%$ of patients were

Table 3. Patient characteristics that influenced outcomes. Profile of married $v s$. not married patients.

$\begin{array}{lccc}\text { Characteristics } & \text { Married } & \text { Single } & \text { P-value } \\ \text { Worked up for DVT/PE } & 5 / 58(8.6 \%) & 10 / 42(23.8 \%) & 0.036 \\ \text { Had helpers at home } & 56 / 59(94.9 \%) & 24 / 43(55.8 \%) & <0.001\end{array}$

Table 4. Patient characteristics that influenced outcomes. Comparing characteristics in patients prescribed warfarin versus patients prescribed other DVT prophylaxis.

\begin{tabular}{|c|c|c|c|}
\hline Characteristics & $\begin{array}{l}\text { Warfarin, } \\
\text { n (\%) }\end{array}$ & $\begin{array}{c}\text { Another DVT prophylaxis, } \\
\text { medication }\end{array}$ & P-value \\
\hline Work-up for DVT/PE & $14 / 68(20.6)$ & $1 / 32(3.1)$ & 0.023 \\
\hline $\begin{array}{l}\text { Required help from surgeon's office } \\
\text { regarding DVT prophylaxis }\end{array}$ & $56 / 69(81.2)$ & $14 / 32(43.8)$ & $<0.001$ \\
\hline Discharged to rehab facility & $41 / 69(59.4)$ & 10/32 (31.3) & 0.008 \\
\hline
\end{tabular}

were found to have one.

Limitations of the study include the geographically distinct area and small sample size. Our small sample size may explain why many variables we measured were not found to correlate with adherence or outcomes. In addition, the six surgeons had varying philosophies and biases influencing their anticoagulation prescribing patterns. Because reports of patient compliance relied on patient's memory, the study results are confounded by recall bias. In addition, the number of non-adherences to DVT medications was relatively small. However, the rates found in our study were consistent with those of previous reports. ${ }^{11,20}$

\section{Conclusions}

This study demonstrates that overall patient compliance with home DVT prophylaxis is high. However, as $17.0 \%$ of patients were noncompliant with DVT prophylaxis medication and a percentage of patients noted that they had a poor understanding of the side effects of the medication, our study supports that there is room to improve patient education. As the patient population for total hip and total knee replacement surgeries continues to grow, DVT prophylaxis adherence will be even more important to emphasize. Future studies will focus on the effects of patient education on improving DVT prophylaxis adherence.

\section{References}

1. Barrack RL. Current guidelines for total joint VTE prophylaxis: dawn of a new day. J Bone Joint Surg Br 2012;94:3-7.

2. Sheth NP, Lieberman JR, Della Valle CJ. DVT prophylaxis in total joint reconstruction. Orthop Clin North Am 2010;41:273-80

3. Hull RD, Pineo GF, Stein PD, et al. Extended out-of-hospital low-molecular-weight heparin prophylaxis against deep venous thrombosis in patients after elective hip arthroplasty: a systematic review. Ann Intern Med 2001;13:85869.

4. Eikelboom JW, Quinlan DJ, Douketis JD. Extended-duration prophylaxis against venous thromboembolism after total hip or knee replacement: a metaanalysis of the randomised trials. Lancet 2001;358:9-15.

5. O'Donnell M, Linkins LA, Kearon C, et al. Reduction of out-of-hospital symptomatic venous thromboembolism by extended thromboprophylaxis with 
low-molecular-weight heparin following elective hip arthroplasty: a systematic review. Arch Intern Med 2003;163: 1362-6.

6. Adelani MA, Keeney JA, Nunley RM, et al. Readmission following total knee arthroplasty: venous thromboembolism as a "never event" is a counterproductive misnomer. J Arthroplasty 2013; 28:747-50.

7. Baba M, Al-Masri M, Salhab M, ElGhanem M. Patient's Compliance on the Use of Extended Low Molecular Weight Heparin Post Major Pelvic Surgeries in Cancer Patients at King Hussein Cancer Center. Gulf J Oncolog 2015;1:73-81.

8. Merkow RP, Bilimoria KY, Sohn MW, et al. Adherence with postdischarge venous thromboembolism chemoprophylaxis recommendations after colorectal cancer surgery among elderly Medicare beneficiaries. Ann Surg 2014;260:103-8.

9. Bellosta R, Ferrari P, Luzzani L, et al. Home therapy with LMWH in deep vein thrombosis: randomized study comparing single and double daily administrations. Angiology 2007;58: 316-22.

10. Bergqvist D, Arcelus JI, Felicissimo P, ETHOS investigators. Post-discharge compliance to venous thromboembolism prophylaxis in high-risk orthopaedic surgery: results from the
ETHOS registry. Thromb Haemost 2012;107:280-7.

11. Deakin DE, Mishreki A, Aslam N, Docker C. Patient compliance with extended low molecular weight heparin injections following hip and knee arthroplasty. Hip Int 2010;20:555-8.

12. Al-Tawfiq JA, Saadeh BM. Improving adherence to venous thromoembolism prophylaxis using multiple interventions. Ann Thorac Med 2011;6:82-4.

13. Cornwell EE 3rd, Chang D, Velmahos $\mathrm{G}$, et al. Compliance with sequential compression device prophylaxis in atrisk trauma patients: a prospective analysis. Am Surg 2002;68:470-3.

14. O'Connor C, Adhikari NK, DeCaire K, Friedrich JO. Medical admission order sets to improve deep vein thrombosis prophylaxis rates and other outcomes. $\mathrm{J}$ Hosp Med 2009;4:81-9.

15. Baskin C, Seetharamu N, Mazure B, et al. Effect of a CD-ROM-based educational intervention on resident knowledge and adherence to deep venous thrombosis prophylaxis guidelines. J Hosp Med 2008;3:42-7.

16. Durieux P, Nizard R, Ravaud P, et al. A clinical decision support system for prevention of venous thromboembolism: effect on physician behavior. JAMA 2000;283:2816-21.

17. Kucher N, Koo S, Quiroz R, et al. Electronic alerts to prevent venous thromboembolism among hospitalized patients. N Engl J Med 2005;352:96977.

18. Brown A. Preventing venous thromboembolism in hospitalized patients with cancer: improving compliance with clinical practice guidelines. Am J Health Syst Pharm 2012;69:469-81.

19. Diaz-Montes TP, Cobb L, Ibeanu OA, et al. Introduction of checklists at daily progress notes improves patient care among the gynecological oncology service. J Patient Saf 2012;8:189-93.

20. Colwell CW Jr, Pulido P, Hardwick ME, Morris BA. Patient compliance with outpatient prophylaxis: an observational study. Orthopedics 2005;28:143-7.

21. Kadakia RJ, Tsahakis JM, Issar NM, et al. Health literacy in an orthopedic trauma patient population: a cross-sectional survey of patient comprehension. J Orthop Trauma 2013;27:467-71.

22. Le Sage S, McGee M, Emed JD. Knowledge of venous thromboembolism (VTE) prevention among hospitalized patients. J Vasc Nurs 2008;26: 109-17.

23. Kneeland PP, Fang MC. Current issues in patient adherence and persistence: focus on anticoagulants for the treatment and prevention of thromboembolism. Patient Prefer Adherence 2010;4:51-60. 